\title{
Towards Landslides Early Warning System With Fog - Edge Computing And Artificial Intelligence**
}

\author{
Meryem Elmoulat $^{a, c}$, Olivier Debauche $e^{b, c, d *}$, Saïd Mahmoudi ${ }^{b}$, Sidi Ahmed Mahmoudi ${ }^{b}$, Adriano \\ Guttadauria $^{b}$, Pierre Manneback $^{b}$, Frédéric Lebeau $^{d}$ \\ ${ }^{a}$ University Mohammed V, Faculty of Sciences, Research Unit GeoRisk: Geological Risks, Battouta Avenue, Rabat, \\ Morocco, 10140 \\ ${ }^{b}$ University of Mons, Faculty of Engineering - ILIA/Infortech, Place du Parc 20, Mons, Belgium, 7000 \\ ${ }^{c}$ University of Liège - GXABT, TERRA, Passage des déportés 2, Gembloux, Belgium, 5030 \\ ${ }^{d}$ University of Liège - GxABT, BioDynE, Gembloux, Belgium, 5030
}

** Data from this paper were partially presented and published in the proceedings of the 11th International Conference on Emerging Ubiquitous Systems and Pervasive Networks (EUSPN) [1].

\begin{abstract}
Landslides are phenomena that cause significant human and economic losses. Researchers have investigated the prediction of high landslides susceptibility with various methodologies based upon statistical and mathematical models, in addition to artificial intelligence tools. These methodologies allow to determine the areas that could present a serious risk of landslides. Monitoring these risky areas is particularly important for developing an Early Warning Systems (EWS). As matter of fact, the variety of landslides' types make their monitoring a sophisticated task to accomplish. Indeed, each landslide area has its own specificities and potential triggering factors; therefore, there is no single device that can monitor all types of landslides. Consequently, Wireless Sensor Networks (WSN) combined with Internet of Things (IoT) allow to set up large-scale data acquisition systems. In addition, recent advances in Artificial Intelligence (AI) and Federated Learning (FL) allow to develop performant algorithms to analyze this data and predict early landslides events at edge level (on gateways). These algorithms are trained in this case at fog level on specific hardware. The novelty of the work proposed in this paper is the integration of Federated Learning based on Fog-Edge approaches to continuously improve prediction models.

Keywords: Landslides Susceptibility, IoT, Artificial Intelligence, Early Warning System, Landslides Monitoring, Edge AI, Edge IoT

\section{Introduction}

Natural disasters are catastrophic events disrupting the ordinary process of Earth, causing millions of life losses and costing billions of economic damages. Among these phenomena, landslides are one of the deadliest events. They occur when the mechanics of the slope movements deteriorate; therefore, the ground transforms into a liquid and flows rapidly and unexpectedly down a steep slope. Furthermore, climate changes intrinsically impact slope instabilities, which increase the landslides' occurrence. The influence of climate changes and landslides have been deeply investigated by our predecessors [27]. Indeed, climate, "its changes", and landslides operate differently and partially on overlapping scales.

Subsequently, there are three main compounds of climate changes that trigger landslides, which are: (1) precipitation, (2) temperature, and (3) weathering. Firstly, changes in precipitation regime can cause erosion and effect land use, which directly impact the equilibrium of vegetation and therefore the slope stability. Secondly, changes in the air temperature influence ice and avalanches, and have indirect impact on rock falls (because of the creation of new fractures within the rocks), and on deep-seated landslides (due to changes in the hydrological cycle). Thirdly, variation in weathering have indirect effect on landslides. A study results of [28] stated that western weather trends caused landslides from autumn to spring and southern patterns were responsible for more landslides in the summer. Hence, the relationship between climate changes and landslides remains complicated to assess and pose serious dilemma to decisions makers and politicians.
\end{abstract}

${ }^{*}$ Corresponding author. Tel.: +3265374059

Meryem Elmoulat and Olivier Debauche are co first authors of this paper.

Fax: +3271140095; E-mail: Olivier.debauche@umons.ac.be

(C) 2021 International Association for Sharing Knowledge and Sustainability.

DOI: $10.5383 / J U S P N .15 .02 .002$ 
For these reasons, it is challenging to detect all areas subject to landslides and to determine when and where they will occur in the future. Knowing areas likely to cause a landslide issue plays a crucial role in terms of regional planning, preservation of human lives, and socio-economic environment. To overcome this problem of landslides susceptibility assessment, prediction, and monitoring, the scientific community used different approaches (statistics, deterministic, mathematical, machine learning...etc.). Nevertheless, these methods depend strongly to the availability of data and intrinsically on their accuracy. For a general approach at various scales and for different types of landslides, these data are often unavailable, but when they are, they present in most of the time insufficient precision to be able to locate the studied phenomenon.

Artificial Intelligence is currently investigated by many research teams to predict displacement of landslides. In explanation, they implement their algorithms on the cloud to train them easily with designated hardware solutions. These researches have proven that artificial intelligence and machine learning algorithms can provide more accurate prediction than classical mathematic and statistic approaches. Nevertheless, the use of AI requires a large database to train algorithms of various kind of landslides to get more robust algorithm.

Nowadays, recent advances in Edge AI provide ability to exploit the power of artificial intelligence at edge level to preprocess data before their transfer to the cloud, in order to train efficient landslides prediction algorithms. Moreover, AI training on the cloud is not adapted for continuous learning, geographical dispersed location, and privacy-sensitive data [15]. Since landslides monitoring is a geoscience matter, means sensing nodes are geographically distributed, and Edge AI computing is well suited to better monitor landslides constraints. In this paper, we investigate the use of AI based on deep neural networks methods to better consider the dynamic nature of landslides and more precisely predict their displacements [29].

The present article will be structured as follow: In section 2, we provide an overview on previous works, summarize landslides susceptibility methods, monitoring factors in early warning systems, data processing at edge level, and finally using artificial intelligence to predict landslides. In section 3, we describe our architecture using a fog micro-cluster, edge gateways on what is deployed as the federated learning algorithms. In section 4, we draw our conclusion. Finally, in section 5, we pave the way to future research directions in section 5 .

\section{Related Works}

In this section, we provide a summary of our main contributions in some of our previous works. Then, we resume the main findings related to this paper.

\subsection{Previous works}

In our previous works, we described the basis of our Wireless Sensor Network (WSN) in [2] and then developed the gateway used to process data near end-users and a multi-agent system [1] We initiated, an edge AI-IoT architecture in [36-37] and described an edge gateway in [38]. In this paper, we propose to evolve these previous works to integrate the federated learning at fog level to continuously improve the prediction accuracy.

\subsection{Landslides Susceptibility Assessment}

Landslides Susceptibility Assessment ( $L S A)$ is a useful tool to predict where landslides may have a higher probability to occur under certain conditions. Over the past decade most researchers in LSA emphasized the use of statistical methods using GIS technologies such as Logistic Regression [29], Weights of Evidence [30], Frequency Ratio [31], etc. Other authors used mathematical approaches to assess landslides susceptibility like Particle Swarm Optimization [12] and Support Vector Machine [9]. Moreover, there are several machines learning techniques that are widely used for landslide spatial prediction such as Decision Tree and Random Forest [6]. Recently, some investigations were conducted to predict landslides zonation by means of Artificial Neural and Network Fuzzy Logic [11,13]. In Table 1, we provide an overview about different susceptibility methods used in the literature. The amount of papers published, these last years, show that Logistic Regression and Frequency Ratio methods are the most used [13].

Table 1. Classification of landslides susceptibility evaluation methods.

\begin{tabular}{|c|c|c|}
\hline Category & Method & Reference \\
\hline \multirow[t]{10}{*}{ Statistical } & Logistic Regression (LR) & {$[6,7,11]$} \\
\hline & Weights of Evidence (WoE) & [6] \\
\hline & Bivariate Statistics (BS) & [7] \\
\hline & Frequency Ratio (FR) & {$[6,7]$} \\
\hline & $\begin{array}{l}\text { Kernel Logistic Regression } \\
(\mathrm{KLR})\end{array}$ & [9] \\
\hline & General Linear Models & [8] \\
\hline & Evidential belief function (EBF) & [13] \\
\hline & Conditional Probability & [13] \\
\hline & Information Value & [13] \\
\hline & Factor Ratio & [13] \\
\hline \multirow[t]{2}{*}{ Mathematical } & $\begin{array}{l}\text { Particle Swarm Optimization } \\
\text { (PSO) }\end{array}$ & [12] \\
\hline & Support Vector Machine (SVM) & {$[9,10,11]$} \\
\hline \multirow[t]{5}{*}{ Decision-aid } & Decision Tree (DT) & [6] \\
\hline & $\begin{array}{l}\text { Multi Criteria Decision Making } \\
\text { (MCDM) }\end{array}$ & [13] \\
\hline & $\begin{array}{l}\text { Analytical Hierarchy Process } \\
\text { (AHP) }\end{array}$ & [7] \\
\hline & $\begin{array}{l}\text { Weighted Linear Combination } \\
\text { (WLC) }\end{array}$ & {$[13]$} \\
\hline & Certainty Factor (CF) & [13] \\
\hline \multirow{2}{*}{$\begin{array}{l}\text { Machine } \\
\text { Learning }\end{array}$} & Random Forest (RF) & [8] \\
\hline & $\begin{array}{l}\text { Classification and Regression } \\
\text { Tree (CRT) }\end{array}$ & [8] \\
\hline \multirow[t]{3}{*}{$\begin{array}{l}\text { Artificial } \\
\text { Intelligence }\end{array}$} & $\begin{array}{l}\text { Artificial Neural Network } \\
(\text { ANN) }\end{array}$ & {$[6,9,11]$} \\
\hline & Fuzzy Logic (FL) & [13] \\
\hline & $\begin{array}{l}\text { Adaptive Network-Based Fuzzy } \\
\text { Inference system (ANFIS) }\end{array}$ & [13] \\
\hline \multirow{4}{*}{$\begin{array}{l}\text { Combination of } \\
\text { methods (i.e.) }\end{array}$} & Logistic Model Tree (LMT) & [9] \\
\hline & Boosted Regression Tree (BRT) & [8] \\
\hline & PSO-ANN & [12] \\
\hline & Bivariate (FR, WoE) - AFNIS & [14] \\
\hline
\end{tabular}

Traditional statistical models assume appropriate structural ones and then focus on parameterizing them. These technics are widely used for analyzing natural hazards such as landslides. We can note that the classification of landslide conditioning factor in traditional statistical models is a key point that affects the quality of landslide susceptibility map. In contrast, machine learning techniques can provide powerful data driven tool, by using algorithms allowing to learn the relationship between a landslide occurrence and landslide related predictors. However, a comparative study of landslide susceptibility maps produced by all the categories of the aforementioned methods in Table 1 (statistical, mathematical, decision-aid, machine learning....etc.) for the same study area and using the same controlling 
parameters, has not been commonly encountered in the literature. For this reason, a comparison among these relatively new approaches is needed to estimate the spatial landslide susceptibility in order to select the best model for regional analysis while taking into account the type of landslides as well as the geomorphological and geological setting of the area being studied. The choice of an adapted susceptibility method to local specificities is crucial for a better monitoring.

\subsection{Early Warning Systems}

We have inventoried early warning systems in our previous work. Interested reader can find more details in Table 1 and more comments in [2]. The main monitoring factors used for the most familiar Early Warning Systems are summarized in Table 2 . The analysis of this table shows that the majority of monitored factor is the rainfall and its derived ones such as infiltration, runoff, run on, and soil moisture, etc.

This major parameter is sometimes associated with earthquake, slope stability, slope angle, or soil wetness.

While other systems use strain evolution, tilt and moisture, or displacement changes.

Table 2. Monitored factors in early monitoring systems.

\begin{tabular}{ll}
\hline Monitored Factors & Reference \\
\hline Rainfall & i.e. [16-17] \\
\hline Rainfall threshold / Slope stability & i.e. [18] \\
\hline Rainfall / Earthquake & i.e. [19] \\
\hline Rainfall / Evaporation & i.e. [20] \\
\hline Strain Evolution & i.e. [21] \\
\hline Tilting angle of slope & i.e. [22] \\
\hline Rainfall / Snowmelt & i.e. [23] \\
\hline Tilt and Moisture & i.e. [24] \\
\hline Displacement Changes & i.e. [25] \\
\hline Rainfall Severity and Soil Wetness & i.e. [26]
\end{tabular}

\subsection{Data processing}

Early Warning System must process data with low latency and react as quickly as possible after the detection of a pattern of landslide triggering. In the literature, we can synthetize different architectural proposition to process data in warning systems.

Abdelaziz et al. [5] have proposed an architecture model for fog computing based on a middleware that abstracts the underlying devices and unifies sensed data, and operational layer to target service presentation, management, and transformation. The proposed architecture has been applied to a flood-warning system. The authors of [3] have suggested a Fog-based architecture that implements various algorithms aiming to schedule tasks of time-sensitive and time-non-sensitive applications with different load balancing policies. This architecture includes four main components: Gateways, the Fog Broker, the cluster of Fog nodes, and applications. Edge gateways ensure the interoperability between sensors protocols, received, aggregated, and preprocessed data transmitted by IoT sensors and devices before their transmission to other tiers. The Fog Broker selects nodes according to the requirement of each application such as: latency and response time throughout processing. Fog cluster supports executing task in containers or virtual machines managed by orchestration framework. Load balancing policies can also be used to share the load between virtual machines. On the other side, Fog nodes composing the fog cluster provide storage and computing resources to edge devices. Finally, they propose a task scheduler which organizes all tasks in the fog cluster.

\subsection{Neural Networks to predict landslides' displacement}

Many authors have implemented Neural Networks to predict landslides' displacement or triggering. We summarize few of these contributions which show convincing results.

Xie et al. [32] demonstrated that Long Short-Term Series with geological conditions, rainfall intensity, and human activities as input factors allow to obtain a better dynamic prediction of displacement than other models.

Zhu et al. [33] developed a multifactor hybrid model with two parts: Least Squares Support Vector Machines (LSSVM) and Double Exponential Smoothing (DES). The model decomposes the one-step ahead in three components: periodic dynamic behavior, trend that represent the geological conditions and, random measuring noise. LSSVM allow to estimate periodic term, while DES calculates the trend component.

Chen et al. [34] evaluated an approach based on Multi Genetic Programming (MGP) by using Separable Functional Network (SFN). However, this method depends of the choice of suitable parameters for Multi-Gene Genetic Programming (MGGP) and the selection of an appropriate structure of Functional Networks (FNs).

Zhou et al. [35] proposed to combine the Wavelet Transform (WT) and Particle Swarm Optimization Kernel Extreme Learning Machine (PSO-KELM) to evaluate landslides displacements. The total displacement is the sum of three component as in [33]: trend, displacement, and noise.

\section{Early Warning System}

In this paper, we propose an adaptable and distributed monitoring system based on an AI-IoT Fog-Edge architecture. The goal of this architecture is to ensure a short delay of treatment with low latency close to users.

This property is particularly important in the context of early warning systems where processing delays and response time are crucial.

Moreover, this architecture allows us also to distribute data preprocessing at gateway level and training at fog level. Both sensors and weather station monitor an area with a high susceptibility level and transmit their data to a common gateway. This data is cleaned and stored at the gateway level for a limited time before their transfer to the micro cluster at Fog Level (See Fig. 1).

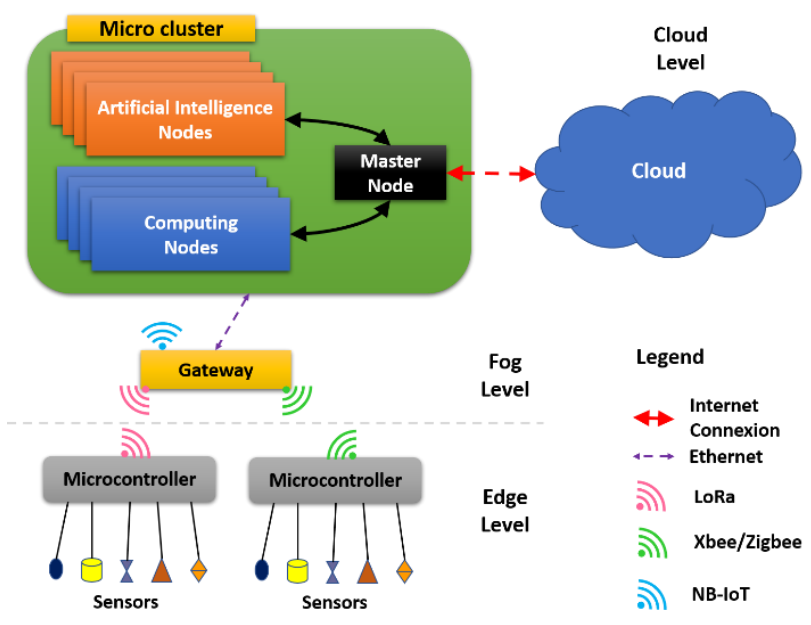

Fig. 1. Architecture of our early warning system

\subsection{Fog micro-cluster}


The intermediary computing units located between the end device and the cloud data center are called fog computing nodes. The fog is composed of all equipment placed between sensors and the cloud such as switch, routers, set-top boxes, and IoT gateways [15].

A micro-cluster is composed of nodes offering better performances thanks to more important capabilities in term of processing, storage, and network than devices and gateways. Hence, the micro-cluster is located at fog level with its capabilities that help us to achieve various processing tasks without having to undergo significant latency times while transferring data to the cloud. The Fog micro cluster provides an interesting settlement between processing capacity and latency. Its characteristics and operation will be detailed in the next section.

\subsubsection{The micro-cluster}

Fog micro-clusters are affordable, easily replicable and extendable, energy efficient and specific to a geographic area [40]. In our architecture, the micro-cluster plays the role of the cloud with a better latency. The micro-cluster is particularly interesting to Landslide Early Warning System to have specific resources dedicated to training AI models near to the sensors and in order to avoid having to transfer data to the cloud to train models.

The Fog-micro cluster that we propose to use in this paper is composed of an association of computing nodes (Raspberry Pi 4B $8 \mathrm{~Gb}$ ) and AI Nodes (Nvidia Jetson Xavier NX). A cluster File System is used to share data between nodes. It is configured in Distributed Volumes based on erasure code that distribute striped encoded data of files across multiple brick in the volume. Moreover, GlusterFS [4] has already been implemented in a fog context in [40] and tested in conjunction with Docker in [39].

In our configuration (Fig. 2), each Raspberry $\mathrm{Pi}$ is equipped of a $500 \mathrm{~Gb}$ SSD except the master node equipped of 1 To SSD connected with a SATA III - USB 3 adaptor. SSD drives of computing nodes are partitioned as follow: $100 \mathrm{~Gb}$ for OS in ext $4,400 \mathrm{~Gb}$ are reserved for the file system Gluster. The master node SSD also contains a supplementary partition of $500 \mathrm{~Gb}$ in the ext 4 filesystem, containing Docker images stored for the local Docker registry. All Raspberry Pi 4 are configured to boot on SSD. Fig. 3 presents the reading speed obtained with SD card, SSD USB3, and SSD NVMe M.2 on Jetson Xavier NX.

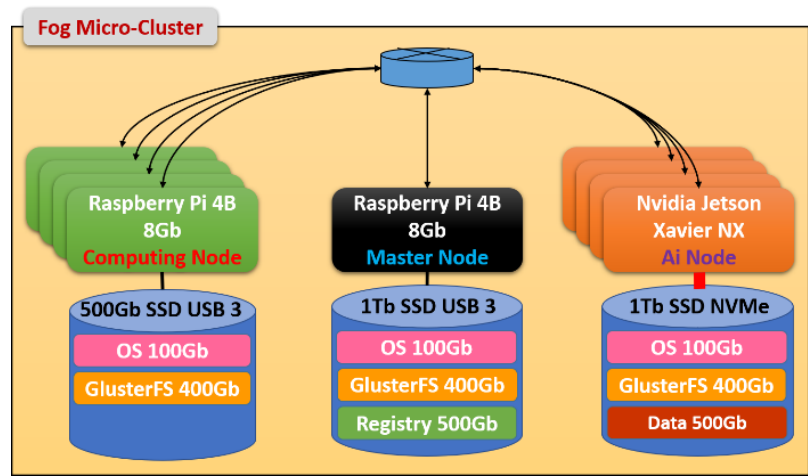

Fig. 2. Fog micro-cluster
On the other side, Jetson Xavier NX nodes that compose our fogmicro-cluster are equipped with 1Tb M.2 NVMe SSD. All nodes are interconnected by means of a 1 Gbits switch. The master node distributed task between other nodes and host the local Docker image registry. GlusterFS is the open-source file system that is deployed on to distribute and store data on the entire cluster.

Table 3. Read Speed on different drives.

\begin{tabular}{clr}
\multicolumn{1}{c}{ Dable 3. Read } & \multicolumn{1}{c}{ Device } & $\begin{array}{c}\text { Reading } \\
\text { Speed [Mb/s] }\end{array}$ \\
\hline SSD USB 3 & Raspberry Pi 4 & 245.3 \\
\hline & Jetson Xavier NX & 268.7 \\
\hline SD Card & Raspberry Pi 4 & 27.8 \\
\hline & Jetson Xavier NX & 28.3 \\
\hline SSD NVMe & Jetson Xavier NX & 564.5 \\
\hline
\end{tabular}

Computing nodes and master node are powered by Ubuntu 20.04 LTS 64 bits for Raspberry Pi 4B 8Gb. While the Nvidia Jetpack 4.4.1 is deployed on Nvidia Jetson Xavier NX. Nvidia Jetson family can cooperate with Azure IoT Edge or AWS IoT Greengrass when more resources are required can run algorithms from different framework while other alternatives such as Edge TPU is only compatible with TensorFlow Lite models and Google Cloud.

The micro cluster can be upgraded by replacing Raspberry Pi 4B by Hardkernel Odroid $\mathrm{H} 2+$, while Nvidia Jetson Xavier NX can be replaced by Nvidia Jetson Xavier AGX in function of needs of computing and/or training.

\subsubsection{IoT Gateways}

IoT gateways support multiples protocols and data formats ensure the interoperability between all sensors. Data received from multiple end-nodes (sensors) are cleaned, aggregated, or fashioned, and stored locally. IoT gateways also ensure data compression and/or preprocessing of data before their transmitting to fog level to advancing processing [3]. Lossless or Lossy data compression offers compression rate up to $10: 1$ and 50:1 respectively [41]. The choice of lossless or lossy data compression algorithm depends on application itself.

Our IoT gateway is a RAK7249 WisGate Edge Max (RAK7249$3 \mathrm{x}-14 \mathrm{x}$ ) that offers 16 LoRa channels, which isequipped with a backup battery and is able to connect multiple backhaul (LTE, Wi-Fi, and Ethernet). An OpenSDK develops custom applications on this gateway powered by OpenWRT.

\subsection{Federated Learning}

This approach is based on AI algorithms for the prediction of landslide triggering and displacement. Broadly speaking, AI training is achieved entirely on the cloud or in an association edge-cloud. However, this paradigm is not adapted for continuous learning, geographically distributed location, and privacy sensitive data, which is the case of landslides warning systems. The Federated Learning (FL) is an emerging distributed learning that distributes training at edge level or endedge-cloud that handles non IID training data, protects privacy, and changes the scale in terms of efficient communication, resource optimization, and security [15].

Indeed, FL helps to deal with non-Independent and Identically Distributed Data (non-IID) training data where each algorithm of AI node works on a portion of data from the complete dataset. Moreover, nodes can have different training capabilities and amount of data to train. Thus, they can contribute variably to the 
global model. FL encompasses this issue thanks to the Federated Averaging of all nodes' contribution to the model update.

In addition to that, IoT nodes present at edge level may undergo from low transmission rate, high latency, or intermittent connections. In fact, IoT nodes are composed of a microcontroller equipped with a set of sensors and powered by a battery or a solar panel. Furthermore, they dispose of very limited processing, storage, and memory capacities. Nevertheless, edge gateways dispose of wider resources than IoT nodes and can be completed with AI accelerators such as GPU and/or TPU.

The training is shared between IoT gateways and fog microcluster (Fig. 3). The cloud is eventually used for the first training of new model on a large amount of data. Then, the model is transferred to fog level where continuous learning is achieved in order to progressively improve the model accuracy and robustness. Edge gateways clear and aggregate data received from IoT nodes.

When the size of the $\mathrm{CNN}$ algorithm to be trained by the micro cluster is too large, it is segmented in a sequence of sub-models. Then, each part of the original CNN is deployed on individual AI nodes to parallelize the training and deal with limited memory. Nonetheless, if the model is small enough to fit in the memory of a node, data can be portioned to parallelize the training.

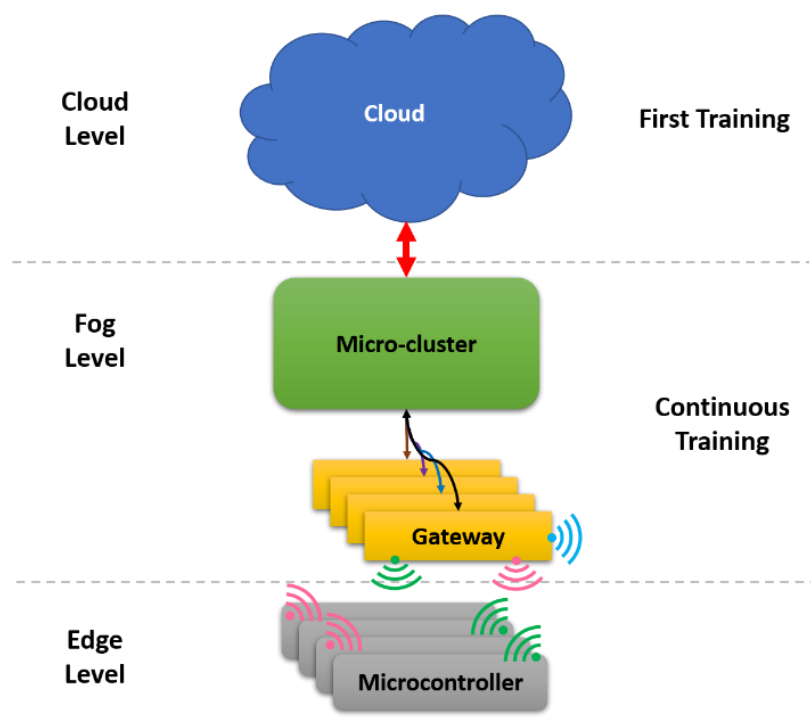

Fig. 3. AI Training process

All AI nodes train the same model simultaneously with its belongings part of the dataset. According to the size of the dataset, the model is shuffled and randomly divided into small parts corresponding to the number of available AI nodes. In the case of larger dataset, model can be trained on chosen samples of the dataset that are trained on AI nodes [15].

Periodically, specific algorithms of artificial intelligences are deployed in the form of docker containers and are then trained on the accumulated data stored on the Fog. After what, Docker containers with retrained algorithms are redeployed by micro Kubernetes (micro k8s) on gateways at edge level. Micro k8s orchestrate containers in the micro-cluster in function of the needs of GPU or not to run the task. The local Docker registry maintain last images of different AI algorithms to allow their rapid deployment. Software stack is used by Ansible to deploy and replicate configuration easily on other new micro-clusters.
Federated Learning (FL) allows us to process locally data without transmission to the cloud. It uses fog micro cluster, deploys near sensors to train models, decrease the latency in term of data transfer, and models the prediction response times. Moreover, FL implements continuous learning in order to improve the accuracy of them instead of having to transfer large amount of data to the cloud to retrain completely models

\section{Conclusion}

In this paper, we have adapted our previous works $[1,2]$ to integrate federated learning at fog level that aims continuously improve prediction accuracy of models. These latter have as main goal to detect anomalies in controlling parameters to predict a potential landslides occurrence by using Federated Learning.

Our first goal was to propose a fog micro-cluster and edge AIIoT architecture capable of adapting the use of various artificial intelligence algorithm adapted to specificities of each landslides, local conditions, and various monitored factors. The second target of this work is to create a flexible architecture in term of integration more powerful hardware and adaptable to treatment's needs without having to change operating system and software stack each time. Moreover, Ansible allows to deploy easily new nodes or redeploy the configuration by using new material more suitable.

In our future works, we will test different intelligence artificial algorithms proposed in the literature and adapt them to be compatible with an operation in edge AI in order to validate definitively our suggested architecture. The latter will be also tested in other contexts than landslides to definitively validate its robustness.

\section{References}

[1] Elmoulat M, Debauche O, Mahmoudi S, Mahmoudi SA, Manneback P, Lebeau F. Edge Computing and Artificial Intelligence for Landslides Monitoring. Proceedings of the $7^{\text {th }}$ International Symposium of Emerging Information, Communication and Networks. Procedia Computer Science 2020; 177: 480-487. https://doi.org/10.1016/j.procs.2020.10.066

[2] Elmoulat M, Debauche O, Mahmoudi S, Ait Brahim L, Manneback P, Lebeau F. Monitoring System Using Internet of Things For Potential Landslides. Proceedings of the The $15^{\text {th }}$ International Conference on Mobile Systems and Pervasive Computing (MobiSPC 2018). Procedia Computer Science 2018; 134: 26-34. https://doi.org/10.1016/j.procs.2018.07.140

[3] Badidi E. QoS-Aware Placement of Tasks on a Fog Cluster in an Edge Computing Environment. Journal of Ubiquitous Systems and Pervasive Networks 2020; 13(1): 11-19. http://doi.org/10.5383/JUSPN.13.01.002

[4] Gluster, Gluster|Storage for your Cloud. Available online: https://www.gluster.org/, (14/01/21)

[5] Abdelaziz J, Adda M, Mcheick H. An Architectural Model for Fog Computing. Journal of Ubiquitous Systems and Pervasive Networks 2018; 10(1): 21-25. https://doi.org/10.5383/JUSPN.10.01.003 
[6] Wang LJ, Guo M, Sawada K, Lin J, Zhang J. A comparative study of landslide susceptibility maps using logistic regression, frequency ratio, decision tree, weights of evidence and artificial neural network. Geoscience Journal 2016; 20: 117-136. https://doi.org/10.1007/s12303-015-0026-1

[7] Yalcin A, Reis S, Aydinogu A, Yomralioglu T. A GISbased comparative study of frequency ratio, analytical hierarchy process, bivariate statistics and logistics regression methods for landslide susceptibility mapping in Trabzon, NE Turkey. CATENA 2011; 85: 274-287. https://doi.org/10.1016/i.catena.2011.01.014

[8] Youssef AM, Pourghasemi HR, Pourtaghi ZS, Al-Katheeri MM. Landslide susceptibility mapping using random forest, boosted regression tree, classification and regression tree, and general linear models and comparison of their performance at Wadi Tayyah Basin, Asir Region, Saudi Arabia. Landslides 2016; 13: 839-856. https://doi.org/10.1007/s10346-015-0614-1

[9] Bui DT, Tuan TA, Klempe H, Pradhan B, Revhaug L. Spatial prediction models for shallow landslide hazards: a comparative assessment of the efficacy of support vector machines, artificial neural networks, kernel logistic regression, and logistic model tree. Landslides 2016; 13 : 361-378.

https://doi.org/10.1007/s10346-015-0557-6

[10] Huang Y, Zhao L: Review on landslide susceptibility mapping using support vector machines. CATENA 2018; 165: 520-529.

https://doi.org/10.1016/j.catena.2018.03.003

[11] Kalantar B, Pradhan B, Naghibi S, Motevalli A, Mansor S: Assessment of the effects of training data selection on the landslide susceptibility mapping: a comparison between support vector machine (SVM), logistic regression (LR) and artificial neural networks (ANN). Geomatics, Natural Hazards and Risks 2018; 9: 46-69. https://doi.org/10.1080/19475705.2017.1407368

[12] Moayedi H, Mehrabi M, Mosallanezhad M, Rashid ASA, Pradham B. Modification of landslide susceptibility mapping using optimized PSO-ANN technique. Engineering with Computers 2019; 35: 967-984. https://doi.org/10.1007/s00366-018-0644-0

[13] Saro L. Current and Future Status of GIS-based Landslide Susceptibility Mapping: A Literature Review. Korean Journal of Remote Sensing 2019; 35(1): 179-193. https://doi.org/10.7780/kjrs.2019.35.1.12 https://doi.org/10.7780/kjrs.2019.35.1.12

[14] Aghdam IN, Pradhan B, Panahi M. Landslide susceptibility assessment using a novel hybrid model of statistical bivariate methods (FR and WOE) and adaptative neurofuzzy inference system (ANFIS) at southern Zagros Mountains in Iran. Environmental Earth Sciences 2017; 76, 237.

https://doi.org/10.1007/s12665-017-6558-0

[15] Wang X, Han Y, Leung VCM, Niyato D, Yan X, Chen X. Edge AI. Convergence of Edge Computing and Artificial Intelligence. Springer: Singapore, 2020. https://doi.org/10.1007/978-981-15-6186-3
[16] Calvello M, Neiva d'Orsi R, Piciullo L, Paes N, Magalhaes $\mathrm{M}$, Alvarenga Lacerda W. The Rio de Janeiro early warning system for rainfall-induced landslides: Analysis of performance for the years 2010-2013. International Journal of Disaster Risk Reduction 2015; 12: 12-15. https://doi.org/10.1016/j.ijdrr.2014.10.005

[17] Piciullo L, Calvello M, Cepeda JM. Territorial early warning systems for rainfall-induced landslides. EarthScience Reviews 2018; 179: 224-247. https://doi.org/10.1016/j.earscirev.2018.02.013

[18] Naidu S, Sajinkumar KS, Oommen T, Anuja VJ, Samuel RA, Muraleedharan C. Early warning system for shallow landslides using rainfall threshold and slope stability analysis. Geoscience Frontiers 2018; 9(6): 1871-1882. https://doi.org/10.1016/j.gsf.2017.10.008

[19] Hong Y, Adler RF. Towards an early-warning system for global landslides triggered by rainfall and earthquake. International Journal of Remote Sensing 2007; 28(16): 3713-3719. https://doi.org/10.1080/01431160701311242

[20] Reder A, Rianna G, Pagano L. Physically based approaches incorporating evaporation for early warning predictions of rainfall-induced landslides. Natural Hazards and Earth System Sciences 2018; 18: 613-631. https://doi.org/10.5194/nhess-18-613-2018

[21] Schenato L, Palmieri L, Camporese M, Bersan S, Cola S, Pasuto A, Galtarossa A, Saladin P, Simonini P. Distributed optical fibre sensing for early detection of shallow landslides triggering. Scientific Reports 2017; 7; 14686. https://doi.org/10.1038/s41598-017-12610-1

[22] Xie J, Uchimura T, Liu J, Xie C, Shen Q. A relationship between displacement and tilting angle of the slope surface in shallow landslides. Landslides 2019; 16: 1243-1251. https://doi.org/10.1007/s10346-019-01135-5

[23] krøgli IK, Devoli G, Colleuille H, Boje S, Sund M, Engen IK. The Norvegian forecasting and warning service for rainfall- and snowmelt-induced landslides. Natural Hazards and Earth System Sciences 2018; 18: 1427-1450. https://doi.org/10.5194/nhess-18-1427-2018

[24] Marciano JS, Hilario CG, Zabanal MAB, Mendoza EV, Gumiran BL, Flores B, Pena MO, Razon KH. Monitoring system for deep-seated landslides using locally-developed tilt and moisture sensors: System improvements and experiences from real world deployment. IEEE Global Humanitarian Technology Conference (GHTC 2014), San Jose, CA, 2014, pp. 263-270. https://doi.org/10.1109/GHTC.2014.6970291 https://doi.org/10.1109/GHTC.2014.6970291

[25] Ma D, Li Y, Cai J, Li B, Li Y, Chen X. Real-Time Diagnosis of Island Landslides Based on GB-RAR. Journal of Marine Science and Engineering 2020; 8(3): 192 https://doi.org/10.3390/jmse8030192

[26] Abraham MT, Satyam N, Pradhan B, Alamri AM. Forecasting of Landslides Using Rainfall Severity and Soil 
Wetnesss: A probabilistic Approach for Darjeeling Himalayas. Water 2020; 12(3): 804. https://doi.org/10.3390/w12030804

[27] Morris B. The components of the Wired Spanning Forest are recurrent. Probab. Theory Rleat Fields 2003; 125: 259265.

https://doi.org/10.1007/s00440-002-0236-0

https://doi.org/10.1007/s00440-002-0236-0

[28] Gariano SL, Guzzeti F. Landslides in a changing climate. Earth-Science Reviews 2016; 162: 227-252. https://doi.org/10.1016/j.earscirev.2016.08.011

[29] Brahim LA, Elmoulat M. Application Of Logistic Regression Method To Produce Landslide Susceptibility Map: A Case Study Of Tetouan Mazari, Morocco. InMATEC Web of Conferences 2018 (Vol. 149, p. 02082). EDP Sciences.

https://doi.org/10.1051/matecconf/201814902082

[30] Elmoulat M, Ait Brahim L. Landslides susceptibility mapping using GIS and weights of evidence model in Tetouan-Ras-Mazari area (Northern Morocco). Geomatics, Natural Hazards and Risk. 2018 Jan 1;9(1):1306-25. https://doi.org/10.1080/19475705.2018.1505666

[31] Elmoulat M, Ait Brahim L. Chapter 2. Landslides Susceptibility Assessment Using Frequency Ratio Method, Remote Sensing Datasets and GIS-Based Techniques. In Krogh DS (Ed), Landslides: Monitoring, Susceptibility and Management. Nova Science Publisher, 2020, pp. 69-114.

[32] Xie P, Zhou A, Chai B. The Application of Long ShortTerm Memory (LSTM) Method on Displacements Prediction on Multifactor-induced Landslides. IEEE Access 2019; 7: 54305-54311. https://doi.org/10.1109/ACCESS.2019.2912419

[33] Zhu X, Xu Q, Tang M, Li H, Liu F. A hybrid machine learning and computing model for forecasting displacement of multifactor-induced landslides. Neural Computing and Applications 2018; 30: 3825-3835. https://doi.org/10.1007/s00521-017-2968-x
[34] Chen J, Zeng Z. Application of multi-gene genetic programming based on separable functional network for landslide displacement prediction. Neural Computing and Application 2016; 27: 1771-1784. https://doi.org/10.1007/s00521-015-1976-y

[35] Zhou C, Yin K, Cao Y, Intrieri E, Ahmed B, Catani F. Displacement prediction of step-like landslide by applying a novel kernel extreme learning machine method. Landslides 2018; 15: 2211-2225. https://doi.org/10.1007/s10346-018-1022-0

[36] Debauche O, Mahmoudi S, Mahmoudi SA, Manneback P, Lebeau P. A new Edge Architecture for AI-IoT services deployment. Procedia Computer Science 2020; 175: 10-19. https://doi.org/10.1016/j.procs.2020.07.006

[37] Debauche O, Mahmoudi S, Elmoulat M, Mahmoudi Sidi Ahmed, Manneback P, Lebeau F. Edge AI-IoT Pivot Irrigation, Plant Diseases, and Pest Identification. Procedia Computer Science 2020; 177: 40-48. https://doi.org/10.1016/j.procs.2020.10.009

[38] Debauche O, Mahmoudi S, Manneback P, Abdessamad A. Fog IoT for Heath: A new Architecture for Patients and Elderly Monitoring. Procedia Computer Science 2019; 160: 289-297. https://doi.org/10.1016/j.procs.2019.11.087

[39] Leite R, Solis P. Performance Analysis of Data Storage in a Hyperconverged Infrastructure Using Docker and GlusterFS. 2019 XLV Latin American Computing Conference (CLEI), Panama, Panama, 2019, pp. 1-10. https://doi.org/10.1109/CLEI47609.2019.235108. https://doi.org/10.1109/CLEI47609.2019.235108.

[40] Cantarutti M. Improving relational database replication with GlusterFS in fog environments. Master Thesis, ING School - School of Industrial and Information Engineering, Polytechnic of Milano; 2018.

https://www.politesi.polimi.it/handle/10589/142836 https://www.politesi.polimi.it/handle/10589/142836

[41] Gia TN, Qingqping L, Queralta P, Zou Z, Tenhunen H, Westerlund T. Edge AI in Smart Farming IoT: CNNs at the Edge and Fog Computing with LoRa. In: 2019 IEEE AFRICON, Accra, Ghana. https://doi.org/10.1109/AFRICON46755.2019.9134049 